\title{
Comparative Analysis of Student Psychology Prediction- Recommendation Two Phase Strategy
}

\author{
Bhakti Ratnaparkhi \\ Department of Information Technology, \\ Pimpri Chinchwad College of Engineering, \\ Pune.
}

\begin{abstract}
Big data analysis includes many theories and methods for prediction system. Statistical methods such as Person's correlation, Regression analysis and Rough Set Theory etc are being used for predicting facts. Also theory like collaboration filtering uses word's filtering to predict and provide recommendations. We have studied all these methods and selected most appropriate method for student's psychology prediction. In our proposed work we have used Rough sets to extract the rules for prediction of student's psychology. Rough Set is a comparatively recent method that has been effective in various fields such as medical, geological and other fields where intelligent decision making is required. Our experiments with rough sets in predicting student's psychology produced attractive results.
\end{abstract}

\section{Keywords}

Student's psychology; prediction; recommendation; rough set theory

\section{INTRODUCTION}

Prediction systems are used to predict future scenarios. E.g. prediction system can be used to in medical domain to predict whether particular disease is present in patient. It has been observed that number of students facing psychological problem is increasing per year. Psychologically disturbed person cannot work efficiently. Same problem is observed in students because of which their educational performance gets affected. To control this situation a prediction system is needed which will accurately predict student's current psychological state.

Recommender system takes help of prediction system to provide recommendations to user. Top ranked predictions are provided as recommendations to user. In real life we take suggestions from our friends or elders because we have trust relation with them. Researchers working in this domain are trying to bring this trust relation in automated systems by increasing accuracy of prediction and recommendations provided from such systems. We need recommender system which will give remedial suggestions to make change in psychological state and improve performance. With pure intension of applying technical knowledge to solve social problem we have proposed our model of prediction \& recommendation approach for student's psychology.

Various methods are available for prediction. By studding these methods we are applying a Rough Set Theory for student's psychology prediction. Rough set theory is useful for rule induction from incomplete data sets. Using this approach we can distinguish between three types of missing attribute values: lost values (the values that were recorded but currently are unavailable), attribute-concept values (these missing attribute values may be replaced by any attribute value limited to the same concept), and "do not care" conditions (the original values were irrelevant). A concept (class) is a set of all objects classified (or diagnosed) the same way. Rough set reduction technique is applied to find all the reducts of the data, which contains the minimal subset of attributes that are associated with a class label for prediction. Finally, rough sets dependency rules are generated directly from all generated reducts.

\section{BACKGROUND STUDY}

\subsection{Various Techniques}

Statistical Method: Manos Papagelis [2] studied many statistics based method and among them selected Pearson's co-relation method to find user with similar interest. Matrix was used to store information related to items and customers as well as group of items. Comparison was made among user based; item based, implicit rating and explicit rating. Here data set used was movie recommendation system, named MRS.

Sakchai Tangwannawit [15] used SVM technique. Data set used was collected from 304 vocational students of the academic year 2012 at Singburi Vocational College.

Rough sets can be use to extract the rules for prediction of personality traits. Rough Set is a recent statistical method that has been used in various fields such as medical, geological and other fields for intelligent decision making. Author performed experiments with rough sets to predict personality traits [17].

Data Mining based Methods: Shuai Zhang [3] developed a system for people with Dementia. Data of 40 patients was collected. Various data mining techniques such as feature selection, feature reduction also different classification algorithms like decision tree (DT), Naïve Bayes (NB) and knearest neighbor $(\mathrm{kNN})$ were applied on it. Results after implementation were analyzed.

Yang Guo [4] used Bayes network for developing a system for people having Type-2 diabetes. Diabetes can be categorized as Type-1 and Type-2. Type- 1 is found in children, which cannot be controlled without insulin. But Type-2 is found in elderly people and it can be controlled without insulin provided it is detected accurately on time. Author developed structure showing dependency of attributes on one another. The data set used was from the UCI Machine Learning Repository. The original owner of this dataset is the National Institute of Diabetes and Digestive and Kidney Diseases.

Bayes network can be used when attributes are dependent on each other. Graph can be use to show dependencies among attributes. Naïve Bayes can be used when attributes are totally independent [7].

Ayman Khedr [5] used FT-tree approach to diagnose Liver disease. The method was very helpful than traditional method 
used for diagnosis of Liver disease. Combination of FT and AT test was used. FT was to detect fibrosis is present or not and AT was to detect how active is the fibrosis. Newly invented method was less painful, it did not required hospitalization plus test was very easy to perform number of times which helps to see the progress of treatment. The dataset contained data on laboratory examinations, which were collected on Electricity Hospital in Egypt.

Samuel and Omisore [6] built a system which was combination of fuzzy system and neural network for prediction of Typhoid. Attributes used were fuzzy in nature to normalize values of fuzzy system author used neural network. Data was collected from hospital administrative.

Apriori algorithm can use to find frequently occurring patterns [8]. This generated patterns can use to recommend products or some important decision making process. In this way Recommender system can use data mining algorithms as Neural Network, Decision Tree, Bayes Network etc [9] [10] [11] [12].

Content based: Content based recommendation is used for recommending news, web sites and books as per user's preference [1] considering user's requirement.

Collaborative Filtering: Collaborative Filtering technique is used in social networking sites to suggest music, jokes [1]. Online shopping websites also use this technique to recommend items that user may want to buy. This technique has some limitations which will be discussed in next section of this document. Now researchers are considering psychological factors as well such as Emotional Intelligence, Satisfaction, Psychological Effect and Personality-based to provide recommendation [1][16].

\subsection{Rough Set Theory}

Rough set theory is a new mathematical approach to imperfect knowledge. The problem of imperfect knowledge has been tackled for a long time by philosophers, logicians and mathematicians. Recently it became also a crucial issue for computer scientists, particularly in the area of artificial intelligence. There are many approaches to the problem of how to understand and manipulate imperfect knowledge. The most successful one is, no doubt, the fuzzy set theory. Rough set theory proposed by the author in [18] presents still another attempt to this problem. The theory has attracted attention of many researchers and practitioners all over the world, who contributed essentially to its development and applications. Rough set theory has an overlap with many other theories. However we will refrain to discuss these connections here. Despite of the above mentioned connections rough set theory may be considered as the independent discipline in its own rights. Rough set theory has found many interesting applications.

The rough set approach seems to be of fundamental importance to AI and cognitive sciences, especially in the areas of machine learning, knowledge acquisition, and decision analysis, knowledge discovery from databases, expert systems, inductive reasoning and pattern recognition. The main advantage of rough set theory in data analysis is that it does not need any preliminary or additional information about data - like probability in statistics, or basic probability assignment in Dempster-Shafer theory, grade of membership or the value of possibility in fuzzy set theory.

In day to day life, we come across with the incomplete or imprecise information or knowledge to understand our surroundings, to learn new things, and to make plans for the future. Rough Set has been introduced by Pawlak in 1982 as a tool to deal with, uncertain knowledge. Its philosophy is based on the assumption that, in contrast to the classical set. This theory uses different approach to uncertainty. The main concept of this Theory is Lower and Upper Approximations. The main advantage of this theory is that it doesn't need any base about data like probability in statistics or grade of membership in fuzzy set theory.

The theory has found many interesting applications in medicine, pharmacology, business, banking, market research, engineering design, conflict analysis, image processing, decision analysis, and other fields. Advantage of this theory is that it allows analyzing both [18]

Due to the imprecision, which exists in real world data, there is sometimes conflicting classification of objects contained in a decision table. The conflicting classification occurs whenever two objects have matching descriptions, but are deemed to belong to different decision classes. In such cases, the decision table is said to contain inconsistencies. Often we wonder whether there are features in the information system, which are more important to the knowledge represented in the equivalence class structure than other features and whether there is a subset of features which by itself can fully characterize the knowledge in the database. Such a feature set is called a reduct. Calculation of reducts of an information system is a key issue in RS theory and we use reducts of an information system in order to extract rule-like knowledge from an information system [20].

\section{PROPOSED WORK}

Following is the model diagram of our proposed model.

In proposed work, first model will be built by learning through data set. Rules will be generated through learning process. Learning will happen only once. With the help of user interface input from student will be taken through Questionnaire which is formed by studding Maslow's motivation theory. These answers will be compared to previously generated rules set. Prediction will be done about student's current psychological state. The predicted state as well as recommendations for improving performance in academics will be provided as an output of complete system to student. Recommendations will be also based on Maslow's Motivation Theory.

For building prediction model two approaches are available data mining and other is statics based approach. We are combing these two approaches to get based possible results. So we call proposed work as 2 phase approach for prediction. In phase 1 , rules will be generated and in phase 2 using statistics current student's data will be matched to some of the rules. 


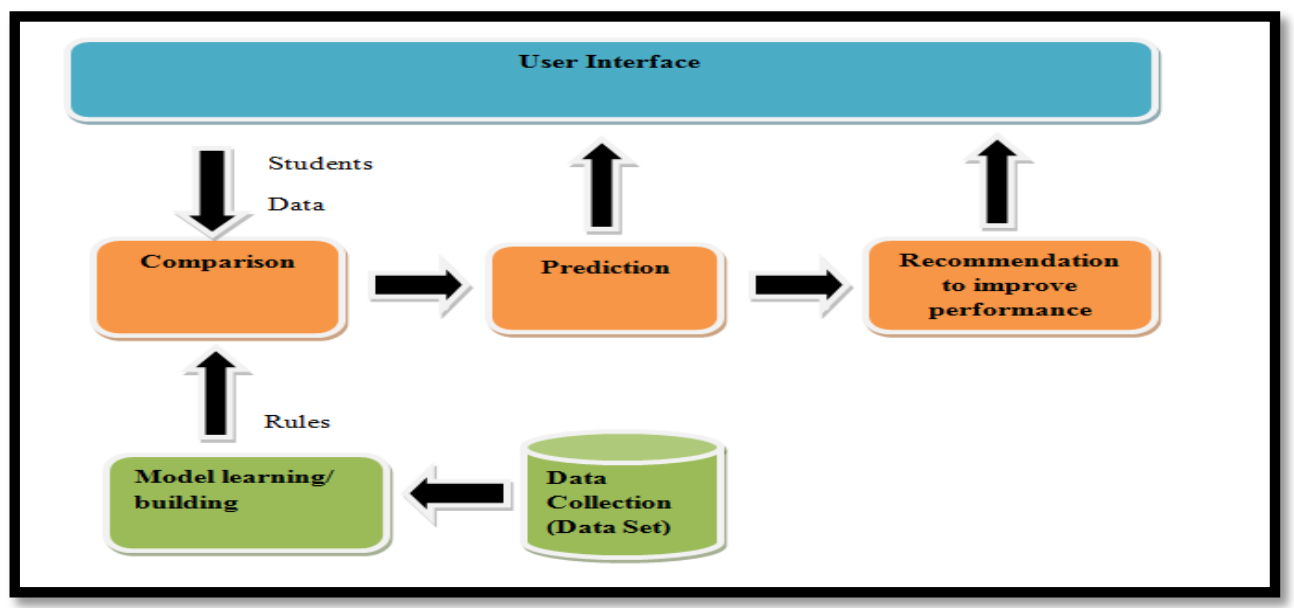

Fig 1: Proposed Model

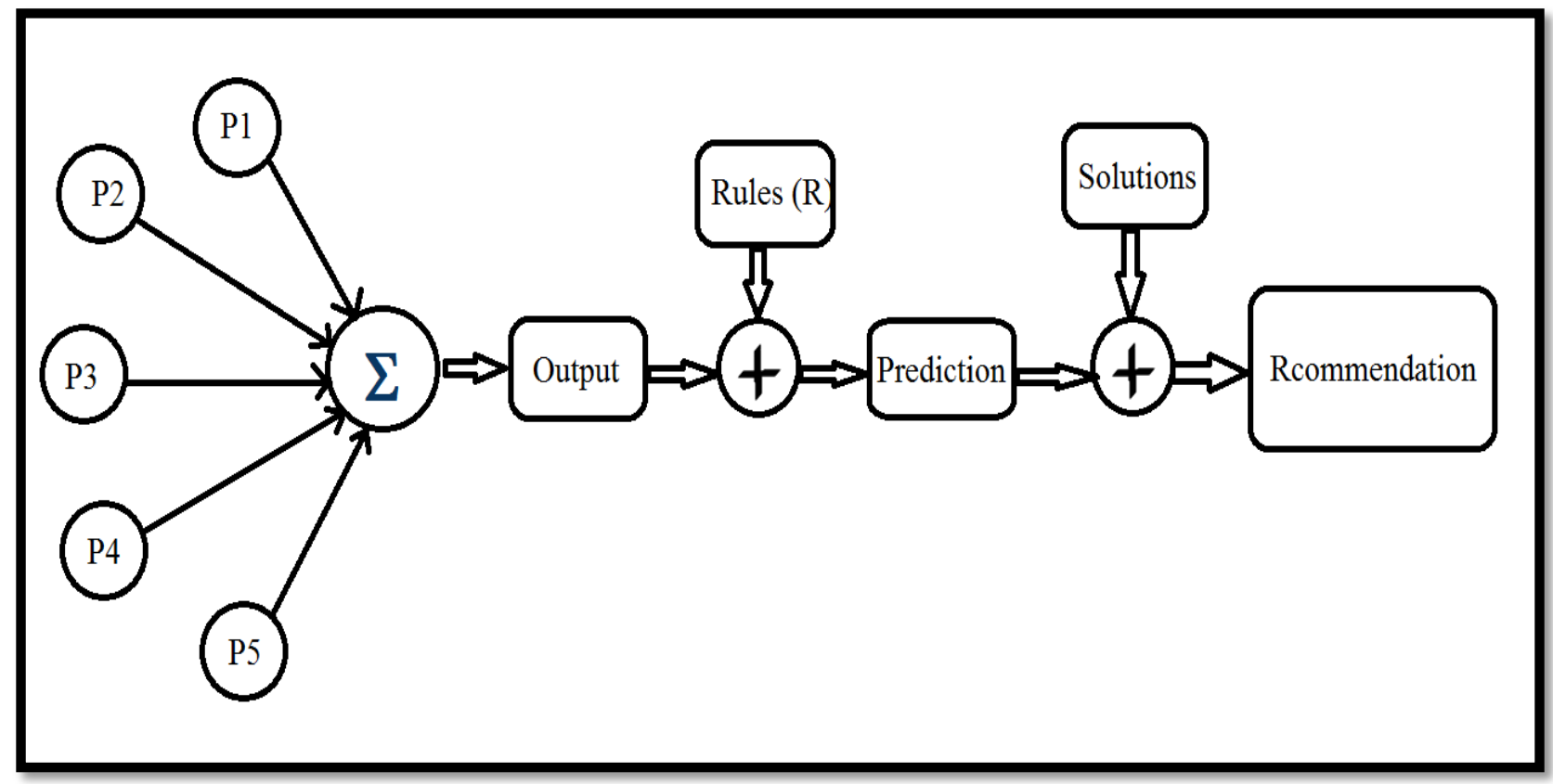

Fig 2: Mathematical Model

$f(p)+R=$ Predictions

$\mathrm{f}(\mathrm{p})=\mathrm{C} 1 \mathrm{p} 1+\mathrm{C} 2 \mathrm{p} 2+\mathrm{C} 3 \mathrm{p} 3+\mathrm{C} 4 \mathrm{p} 4+\mathrm{C} 5 \mathrm{p} 5=\Sigma \mathrm{p}$

$\mathrm{p} 1$, p2 etc are parameters based on which student's psychology will be judged. $\mathrm{f}(\mathrm{p})$ as shown in above equation is aggregation of all these parameters of individual student. $\mathrm{R}$ is a set of rules which will be formed by learning through data set. Current student's parameters will be then compared to set of rules i.e. $\mathrm{R}$ and prediction will be done. Here C1, C2, C3, $\mathrm{C} 4, \mathrm{C} 5=$ constants considered as per weight.

\section{IMPLEMENTATION SUPPORT}

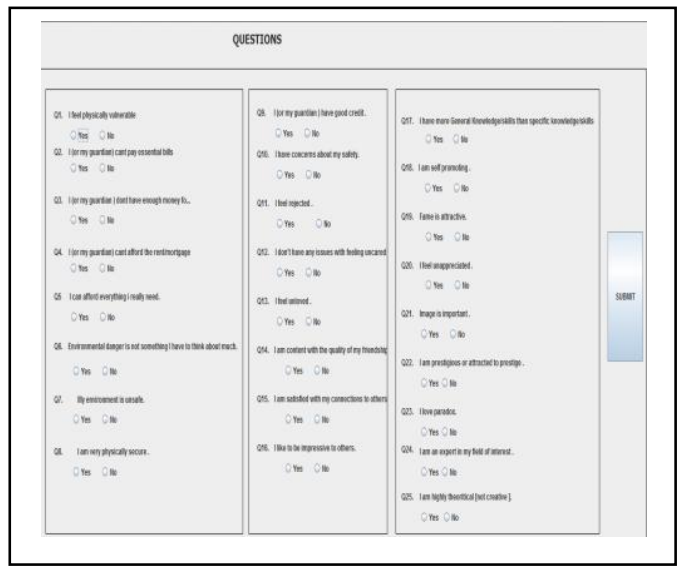

Fig 3: Questionnaire

To demonstrate proposed model we have performed simulation of our idea. Questionnaire was formed on five parameters as shown in mathematical model based on 
Maslow's theory. Student will submit response by clicking correct option for every question. Then prediction report of his psychology will be generated as shown in Fig. 4 bellow.

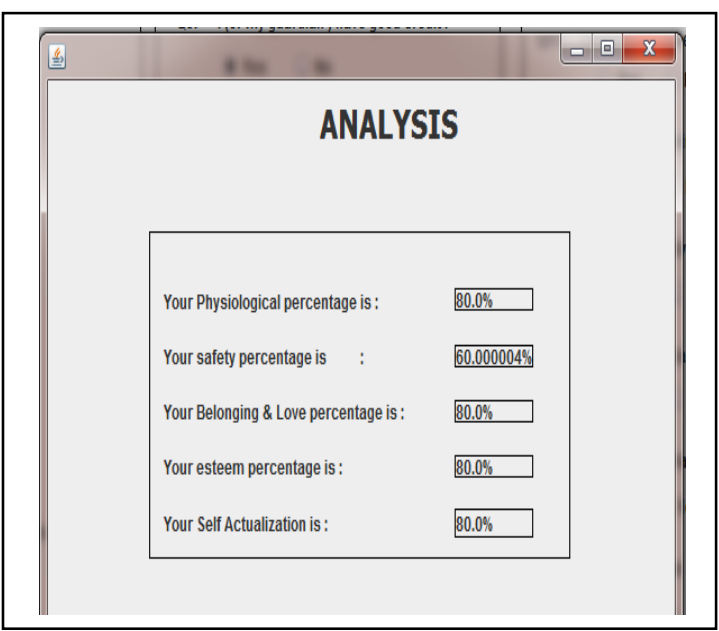

Fig 4: Prediction Report

\section{RESULTS}

Accuracy $=($ Lower approximation $) /$ (Upper approximation). As 9,10 is case of uncertainty, accuracy should be calculated for only these 2 cases. For all other cases as there is no conflict in decision accuracy will be $100 \%$. For case 9; lower approximation (for $D=5$ ) is 1 and upper approximation is $2+1$ so accuracy will be $(1 / 3)=0.33$ i.e. $33.33 \%$. For case 10 ; lower approximation (for $\mathrm{D}=2$ ) is 5 and upper approximation is $5+2$ so accuracy will be $(5 / 7)=0.71$ i.e. $71.4 \%$. In Fig. 5 . We can see comparison of proposed method to SVM.

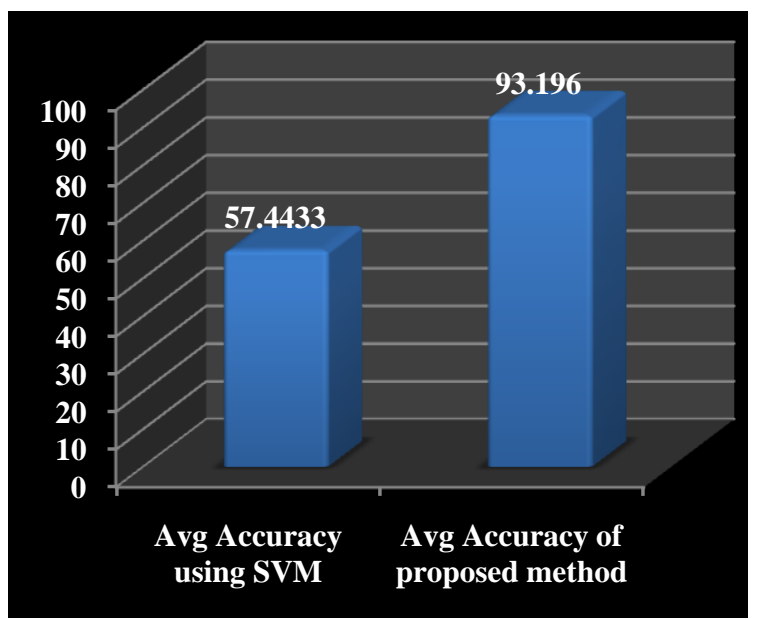

Fig 5: Comparative Result

\section{CONCLUSION}

Various techniques used for prediction and recommender system were studied in order to develop system for prediction of student psychology and provide remedial actions to improve their academic performance with improved accuracy in prediction and recommender system.

Maslow motivation theory was also studied which will be used for predicting current state of student's psychology and also to provide suggestions for improvements. Our own 2phase model was proposed and results were obtained through simulation.

\section{REFERENCES}

[1] Maria Augusta S. N. Nunes, "Towards To PsychologicalBased Recommenders Systems: A Survey on Recommender Systems", Scientia Plena Vol. 6, Num. 8 2010.

[2] Manos Papagelis, DimitrisPlexousakis, IoannisRousidis and Elias Theoharopoulos, "Qualitative Analysis of Userbased and Item-based Prediction Algorithms for Recommendation Systems".

[3] Shuai Zhang, Sally I. Mcclean, "A Predictive Model for Assistive Technology Adoption for People With Dementia", Ieee Journal Of Biomedical And Health Informatics, Vol. 18, No. 1, January 2014.

[4] Yang Guo, GuohuaBai, Yan Hu, "Using Bayes Network for Prediction Of Type-2 Diabetes", 2012, Ieee, 7th International Conference For Internet Technology And Secured Transactions (Icitst).

[5] AymanKhedr,"Business Intelligence Framework To Support Chronic Liver Disease Treatment", International Journal Of Computers \& Technology Volume 4 No. 2, March-April, 2013, Issn 2277-3061.

[6] Samuel and Omisore, "Hybrid Intelligent System for the Diagnosis of Typhoid Fever", J ComputEngInfTechnol 2013, 2:2, Journal of Computer Engineering \& Information Technology.

[7] "Diagnosis of Heart Disease for Diabetic Patients using Naive Bayes Method", International Journal of Computer Applications (0975 - 8887) Volume 24- No.3, June 2011.

[8] "Finding Locally Frequent Diseases Using Modified Apriori Algorithm", International Journal of Advanced Research in Computer and Communication Engineering Vol. 2, Issue 10, October 2013.

[9] "Importance of Artificial Neural Network in Medical Diagnosis disease like acute nephritis disease and heart disease", International Journal of Engineering Science and Innovative Technology (IJESIT) Volume 2, Issue 2, March 2013.

[10] "Lung cancer differential diagnosis based on the computer assisted radiology: The state of the art"

[11] "The Application of Machine Learning Technique for Malaria Diagnosis"

[12] "Performance Evaluation of Levenberg-Marquardt Technique in Error Reduction for Diabetes Condition Classification", International Conference on Computational Science, ICCS 2013.

[13] "An Investigation into the Feasibility of Detecting Microscopic Disease Using Machine Learning", Keynote Lecture of IEEE International Conference on Bioinformatics and Biomedicine November 2-4, 2007, Silocon Valley, California, USA.

[14] ArturasKaklauskas, EdmundasKazimierasZavadskas, VaidotasTrinkunas, Laura Tupenaite, Justas Cerkauskas, PauliusKazokaitis, "Recommender system to research students' study efficiency", Procedia - Social and Behavioral Sciences 51 ( 2012 ) 980 - 984.

[15] SakchaiTangwannawit and MonteanRattanasiriwongwut, "Comparing the Strengths and Difficulties Questionnaire 
(SDQ) and Behavior Consideration Assessment Using SVM Techniques", DOI: 10.7763/IPEDR. 2014. V70. 16.

[16] Bhakti Ratnaparkhi, Prof. Dr.J. S. Umale, "State of the art of Prediction and Recommender System", International Journal of Computer Applications (0975 8887) Volume 108 - No. 11, December 2014.
[17] Umang Gupta, Niladri Chatterjee, "Personality Traits Identification using Rough sets based Machine Learning”, IEEE 2013 International Symposium on Computational and Business Intelligence.

[18] Bhakti Ratnaparkhi, Dr. J. S. Umale, "Improved student psychology prediction \& recommendation strategy using 2 state data analysis", IEEE Global Conference on Communication Technologies 2015. 